\title{
Modulation of mitogen-activated protein kinase attenuates sepsis-induced acute lung injury in acute respiratory distress syndrome rats
}

\author{
WEI FANG ${ }^{*}$, SHI-XIA CAI ${ }^{*}$, CHUAN-LEI WANG, XIAO-XIA SUN, KUN LI, XIAO-WEN YAN, YUN-BO SUN, \\ XIAO-ZHE SUN, CHUAN-KAI GU, MING-YING DAI, HUI-MING WANG and ZHEN ZHOU
}

Department of Critical Care Medicine, The Affiliated Hospital of Qingdao University, Qingdao, Shandong 266003, P.R. China

Received January 4, 2017; Accepted August 10, 2017

DOI: $10.3892 / \mathrm{mmr} .2017 .7811$

\begin{abstract}
Sepsis is the most important predisposing cause inducing acute respiratory distress syndrome (ARDS); however, the mechanism of sepsis leading to the development of ARDS remains to be elucidated. Suppression of the mitogen-activated protein kinase (MAPK) signal by blocking the phosphorylation of Jun N-terminal kinase (JNK) and p38 in lung tissues could alleviate acute lung injury induced by sepsis. MAPK signaling may have a crucial role in development of the sepsis-induced acute lung injury. The specific inhibitors of JNK and p38 MAPK, SP600125 and SB203580, were administrated by intragastric injection $4 \mathrm{~h}$ before induction of ARDS after cecal ligation and puncture (CLP). Rats were sacrificed at 1,6 or $24 \mathrm{~h}$ after CLP challenge. The histological evaluation, lung water content, and biochemical analysis were performed. The results revealed that the JNK and p38 MAPK inhibitor improved lung permeability, attenuated system inflammation, further alleviated the lung injury induced by sepsis. In
\end{abstract}

Correspondence to: Dr Shi-Xia Cai, Department of Critical Care Medicine, The Affiliated Hospital of Qingdao University, 16 Jiangsu Road, Qingdao, Shandong 266003, P.R. China

E-mail: cccsssxxx1980@163.com

*Contributed equally

Abbreviations: ARDS, acute respiratory distress syndrome; MAPK, mitogen-activated protein kinase; JNK, Jun N-terminal kinase; CLP, cecal ligation and puncture; ICU, intensive care unit; ERK, extracellular signal-regulated kinase; TLR4, Toll-like receptor 4; SD, Sprague-Dawley; DMSO, dimethyl sulfoxide; LWC, lung water content; RIPA, radioimmuneprecipitation assay; SDS-PAGE, sodium dodecyl sulfate-polyacrylamide gel electrophoresis; PVDF, polyvinylidene fluoride; TBS, Tris-buffered saline; HRP, horseradish peroxidase; H\&E, hematoxylin and eosin; W/D, wet/dry weight ratio; IL-6, interleukin-6; IL-10, interleukin-10; TNF- $\alpha$, tumor necrosis factor- $\alpha$; ELISA, enzyme-linked immunosorbent assay

Key words: MAPK, JNK, p38 MAPK, sepsis, acute lung injury, acute respiratory distress syndrome conclusion, JNK and p38 MAPK signaling are essential for the development of ARDS following sepsis. Further studies are needed to illuminate the detailed mechanisms of JNK and p38 MAPK signaling in sepsis-induced ARDS.

\section{Introduction}

Acute respiratory distress syndrome (ARDS) is acute respiratory failure, which was the main cause of death among critically ill patients, with a mortality rate as high as $40 \%(1,2)$. ARDS has a close association with sepsis in the intensive care unit (ICU). Sepsis is the most common of ARDS (3), $~ 50 \%$ of ICU patients with sepsis also have complications with ARDS (4). Additionally, clinical research reveals that when sepsis-induced ARDS patients are compared with non-sepsis ARDS patients, greater severity of the illness and higher in-hospital mortality rates were observed (5). However, the mechanism of the sepsis leading to the development of ARDS remains to be elucidated (6).

Sepsis is the systematic response to infection; excessive inflammation damages the lungs leading to ARDS. Previous studies show inflammatory damage triggers a robust influx of neutrophils and monocytes to the site of tissue injury (7), and the damaged or dead cells may trigger the inflammasome-dependent responses, then alert the innate immune system to the impending tissue damage, suggesting differing roles for inflammasomes in the development of ARDS (8). The mitogen-activated protein kinase (MAPK) signal transduction pathways of inflammatory cells modulate a dysregulated, overly aggressive inflammatory response, which promotes the development of sepsis (9). Inflammatory signals are initiated by the recognition of inflammatory stimuli by specific transmembrane and intracellular receptors, previous studies have found that following inflammatory stimulation, some major MAPK subfamilies, including extracellular signal-regulated kinase (ERK), p38 and Jun N-terminal kinase (JNK) were activated (10), which induces the expression of multiple genes that together regulate the inflammatory response (11).

Through the phosphorylation of a range of downstream substrates, different MAPKs have diverse roles in transmitting the receptor-proximal signals to the transcriptional activation of selected genes (12). Previous studies found that MAPK 
pathways may provide drug targets in inflammation to inhibit cytokine production $(13,14)$. Previous studies determined that in mice with cecal ligation and puncture (CLP) the protein expression of Toll-like receptor 4 (TLR4), phosphorylated (p)-p38, p-JNK and p-ERK was increased, whereas treatment with Compound 9a protected against septic injury by suppressing MAPK-mediated inflammatory signaling (15). In a lipopolysaccharide (LPS)-stimulated ARDS mouse model, some drugs or compounds (including Decitabine, 5-azacitidine, Andrographolide sulfonate, Hydroxy-Jolkinolide B-1 and Astilbin) alleviated LPS-induced ARDS by suppressing LPS-induced activation of the MAPK signaling pathways by blocking the phosphorylation of JNK, ERK and p38 in lung tissues (16-19). Previous studies indicated that glycyrrhizic acid and Losartan have a protective effect against sepsis-induced acute lung injury by inhibiting the inflammatory response, reducing damage from oxidative stress, and apoptosis via inactivation of JNK and p38 MAPK $(20,21)$. The aforementioned studies indicated the effect of MAPK signaling, particularly the JNK and p38 MAPK on sepsis-induced ARDS. The aim of the current study was to identify the effect of MAPK signaling on sepsis-induced acute lung injury in ARDS rats, to further clarify the mechanism of sepsis leading to the development of ARDS.

\section{Materials and methods}

Animals. A total of 72 adult male Sprague-Dawley (SD) rats (6-8 weeks old; weight, 220-270 g) were obtained from Laboratorial Animal Center of Shandong University (Jinan, China). All animals were kept in a standard environment with $\sim 23^{\circ} \mathrm{C}$ room temperature, $30-60 \%$ humidity and a $12-\mathrm{h}$ light/dark cycle, allowed free access to standard rodent chow and drink, and adapted to laboratory conditions for a minimum of 3 days. The study protocols conformed to the Guide for the Care and Use of Laboratory Animals (National Institutes of Health, Bethesda, MD, USA) and were approved by the Institutional Animal Care and Use Committee of Qingdao University (Qingdao, China).

Rat model of sepsis-induced ARDS (22). All rats were anesthetized with $10 \%$ chloral hydrate $(300 \mathrm{mg} / \mathrm{kg})$ by intra-peritoneal injection before surgical procedures with $8 \mathrm{~h}$ preoperative fasting food. After a $2-\mathrm{cm}$ incision through midline, the cecum was carefully isolated and ligated at distal to the ileocecal valve with a $4-0$ silk suture to avoid intestinal obstruction. The cecum was punctured twice with a sterile 20 -gauge needle and gently squeezed to extrude a small amount of feces from the perforation sites. The quantity of extruded feces was limited (small droplet, $\sim 1 \mathrm{~mm}$ in diameter) and consistent in all rats. Next, the abdominal cavity was closed in two layers with continuous suture of 3-0 silk after the cecum was returned. In the sham group, the abdomen was opened and the cecum manipulated, but no cecal ligation or cecal puncture was performed and the abdomen was closed. Following surgery each rat received $1 \mathrm{ml}$ normal saline by subcutaneous injection for fluid resuscitation and no antibiotics were administrated.

Experimental protocol. Rats were randomly divided into 6 groups according to a random number table $(n=12$ for each group): i) Sham group (group A); ii) ARDS group (group B), in which rats were challenged with CLP to induce the ARDS model; iii) dimethyl sulfoxide (DMSO) + ARDS group (group C), rats received an equal amount of 10\% DMSO $4 \mathrm{~h}$ before induction of ARDS (16); iv) SP600125 + ARDS group (group D), rats received SP600125 (JNK inhibitor) at $30 \mathrm{mg} / \mathrm{kg}$ $4 \mathrm{~h}$ before induction of ARDS, SP600125 was dissolved in 10\% DMSO; v) SB203580 + ARDS group (group E), rats received SB203580 (p38 MAPK inhibitor) at $10 \mathrm{mg} / \mathrm{kg} 4 \mathrm{~h}$ before induction of ARDS, SB203580 being dissolved in DMSO; and vi) SP600125 + SB203580 + ARDS group (group F), rats received SP600125 (30 mg/kg) and SB203580 (10 mg/kg), $4 \mathrm{~h}$ before induction of ARDS. DMSO (Amresco, LLC, Solon, OH, USA), SP600125 (Selleck Chemicals, Houston, TX, USA) and SB203580 (Selleck Chemicals) were all administered by intragastric injection, in group A and group B rats received an equal amount of normal saline. Rats were sacrificed at 1, 6 or $24 \mathrm{~h}$ after CLP challenge, and samples were collected from each rat for histological evaluation, lung water content (LWC) $\mathrm{t}$ and biochemical analyses.

Western blot analysis. Total protein was extracted by using radioimmunoprecipitation assay (RIPA) lysis buffer (Beyotime Institute of Biotechnology, Haimen, China). Protein was separated by $10 \%$ sodium dodecyl sulfate-polyacrylamide gel electrophoresis (SDS-PAGE) and electro-transferred to polyvinylidene fluoride (PVDF) membranes $(0.45 \mathrm{~mm}$; EMD Millipore, Bedford, MA, USA). The membranes were blocked in Tris-buffered saline (TBS; $\mathrm{pH}$ 7.4) containing $0.1 \%$ Tween-20 (Shanghai Chemical Reagent Company of China Pharmaceutical Group, Shanghai, China) and 5\% bovine serum albumin (Thermo Fisher Scientific, Inc, Waltham, MA, USA) for $1 \mathrm{~h}$ at room temperature, then incubated at $4^{\circ} \mathrm{C}$ overnight with primary antibodies for p-JNK (cat. no. sc-135642; 1:100), total JNK (cat. no. sc-571; 1:100), p-p38 MAPK (cat. no. sc-101759; 1:100), total p38 (cat. no. sc-7149; 1:100) and $\beta$-actin (cat. no. sc-130656; 1:500; all from Santa Cruz Biotechnology, Inc, Dallas, TX, USA). Subsequently, membranes were incubated for $1 \mathrm{~h}$ at room temperature with a goat anti-rabbit IgG-horseradish peroxidase (HRP)-conjugated secondary antibody (cat. no. 93974; 1:10,000; OriGene Technologies, Beijing, China). Immunoreactive bands were detected with Pierce ECL western blotting substrate (Thermo Fisher Scientific, Inc.).

Hematoxylin and eosin $(H \& E)$ staining and lung injury scoring. The right upper lobe of the lung was embedded in paraffin (Thermo Fisher Scientific, Inc.) and sectioned at $5 \mu \mathrm{m}$. The sections were stained with H\&E (Beyotime Institute of Biotechnology). The severity of lung injury was determined as previously described (23). Lung injury was graded from 0 (normal) to 4 (severe) for the following: Edema, alveolar and interstitial inflammation, alveolar and interstitial hemorrhage, atelectasis and hyaline membrane formation. The total lung injury score per mouse was determined as sum of the aforementioned scores. Two investigators blinded to the experimental protocol analyzed ten randomly selected high-power fields in each slide at a magnification $\mathrm{x} 400$.

Evaluation of the LWC. The right lower lobe of the lung from the rats was weighed immediately and subsequently 

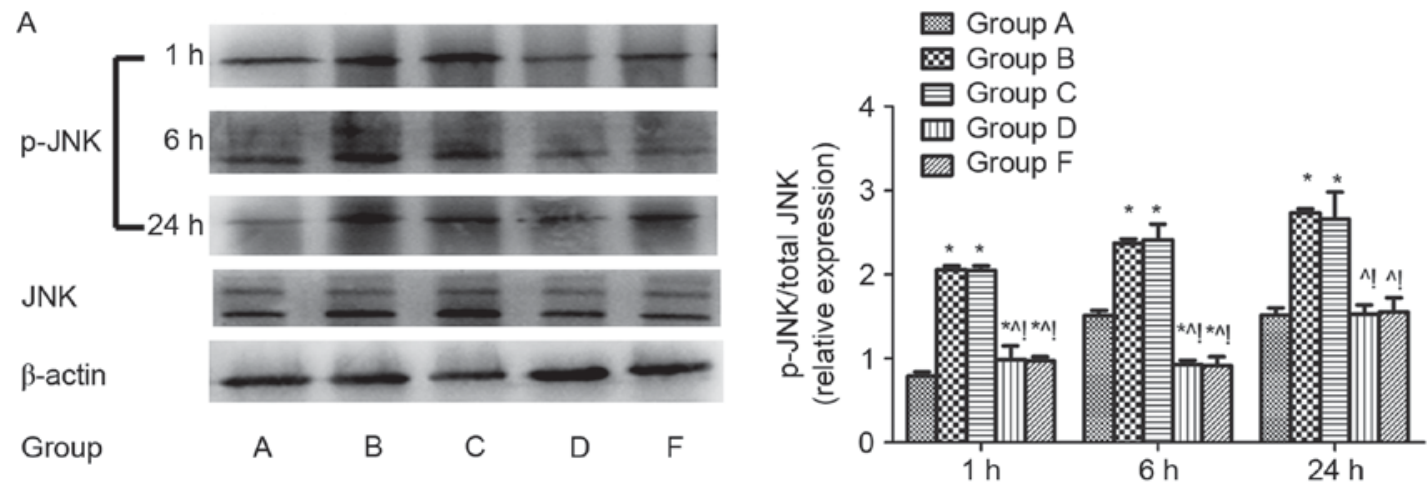

B

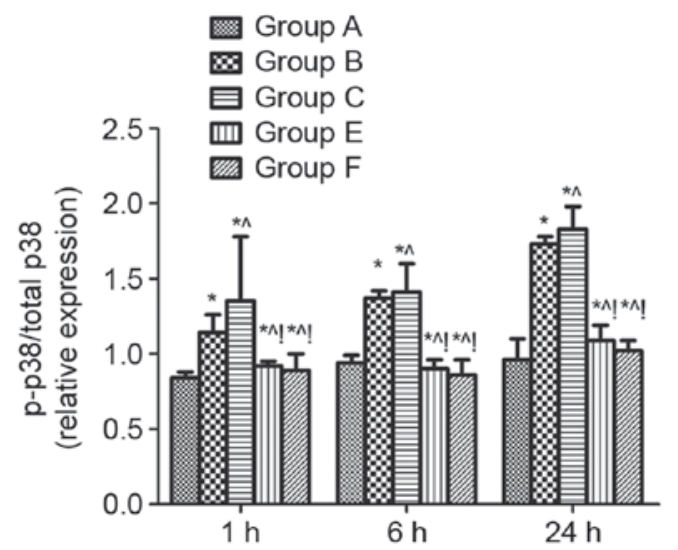

Figure 1. Regulation of JNK and p38 MAPK signalling in lungs after cecal ligation and puncture challenge. The regulation of pulmonary JNK and p38 MAPK signalling were assayed by ratio of (A) phosphorylated JNK/total JNK and (B) phosphorylated p38/total p38 in the lung tissue 1, 6, and $24 \mathrm{~h}$ after CLP challenge analyzed by western blotting. $\beta$-actin was used as an internal control, and the results were expressed as mean \pm standard deviation. $n=4$ at each time point for each group. ${ }^{*} \mathrm{P}<0.05$ vs. group $\mathrm{A},{ }^{\wedge} \mathrm{P}<0.05$ vs. $\mathrm{B},{ }^{\prime} \mathrm{P}<0.05$ vs. $\mathrm{C}, \mathrm{D}, \mathrm{E}$ and $\mathrm{F}$. ARDS, acute respiratory distress syndrome; MAPK, mitogen activated protein kinase; JNK, Jun N-terminal kinase; A, sham; B, ARDS; C, DMSO + ARDS; D, SP600125 + ARDS; E, SB203580 + ARDS; F, SP600125 + SB203580 + ARDS.

dried at $80^{\circ} \mathrm{C}$ for $48 \mathrm{~h}$ to calculate the wet/dry weight ratio (W/D). The LWC was calculated using the following formula: $\mathrm{LWC}=(1-\mathrm{W} / \mathrm{D}) \times 100$.

Cytokine detection. Blood samples $(5 \mathrm{ml})$ were collected by cardiac puncture and centrifuged at $1,500 \mathrm{x}$ g for $5 \mathrm{~min}$ at $4^{\circ} \mathrm{C}$. The concentration of interleukin- 6 (IL-6), IL-10, and tumor necrosis factor- $\alpha(\mathrm{TNF}-\alpha)$ proteins in the supernatant were detected using a commercial rat cytokine-specific enzyme-linked immunosorbent assay (ELISA) kits (cat. nos. MM-0190R1, MM-0195R1 and MM-0180R1; Jingmei Biotech, Beijing, China) following the manufacturer's protocol. All samples were tested in duplicate.

Statistical analysis. The data were presented as the mean \pm standard deviation. Statistical analyses were performed using SPSS version 19.0 (IBM, Armonk, NY, USA). Comparisons among multiple groups were performed using one-way ANOVA followed by a Bonferroni's post hoc test. $\mathrm{P}<0.05$ was considered to indicate a statistically significant difference.

\section{Results}

Regulation of pulmonary JNK and p38 MAPK signalling after CLP challenge. The protein expression of total JNK, total p38 MAPK, p-JNK and p38 MAPK (p-p38 MAPK) in lung tissues 1,6 , and $24 \mathrm{~h}$ after CLP challenge were evaluated using western blotting analysis. The p-JNK/total JNK and p-p38 MAPK/total p38 MAPK protein were increased in the sepsis-induced lung injury group (group B) compared with the shame control group (group A) $(\mathrm{P}<0.05)$. The $\mathrm{p}-\mathrm{JNK} /$ total JNK protein was downregulated in the groups $\mathrm{D}$ and $\mathrm{F}$ compared with group $\mathrm{B}(\mathrm{P}<0.05$; Fig. 1A) and the p-p38 MAPK/total p38 MAPK protein was downregulated in the group $\mathrm{E}$ and $\mathrm{F}$ compared with group $\mathrm{B}$ $(\mathrm{P}<0.05$; Fig. 1B). The same results were found when compared with the DMSO control (group $\mathrm{C})(\mathrm{P}<0.05$; Fig. 1).

Effect of MAPK signalling on pulmonary histopathology of sepsis-induced acute lung injury rats. The thickening of the alveolar wall, alveolar and interstitial inflammatory cell infiltration, haemorrhaging, alveolar exudates and the edema were increased in the lung tissue of rats after sepsis-induced lung injury (group B), and the lung injury score for quantification of the lung injury was also increased compared with group A $(\mathrm{P}<0.05)$. However, these histopathological characteristics and the lung injury score were alleviated at 1,6 , and $24 \mathrm{~h}$ in the groups $\mathrm{D}, \mathrm{E}$ and $\mathrm{F}$ compared with group $\mathrm{B}(\mathrm{P}<0.05)$. The same effect was found when compared with the DMSO control (group C) $(\mathrm{P}<0.05$; Fig. 2).

Effect of MAPK signalling in sepsis-induced lung permeability. The LWC was calculated to evaluate lung edema. LWC was significantly reduced at 1,6 , and $24 \mathrm{~h}$ in the groups $\mathrm{D}$, $\mathrm{E}$ and $\mathrm{F}$ compared with group $\mathrm{B}(\mathrm{P}<0.05)$. The same effect was found when compared with the DMSO control (group C) ( $\mathrm{P}<0.05$; Fig. 3). 


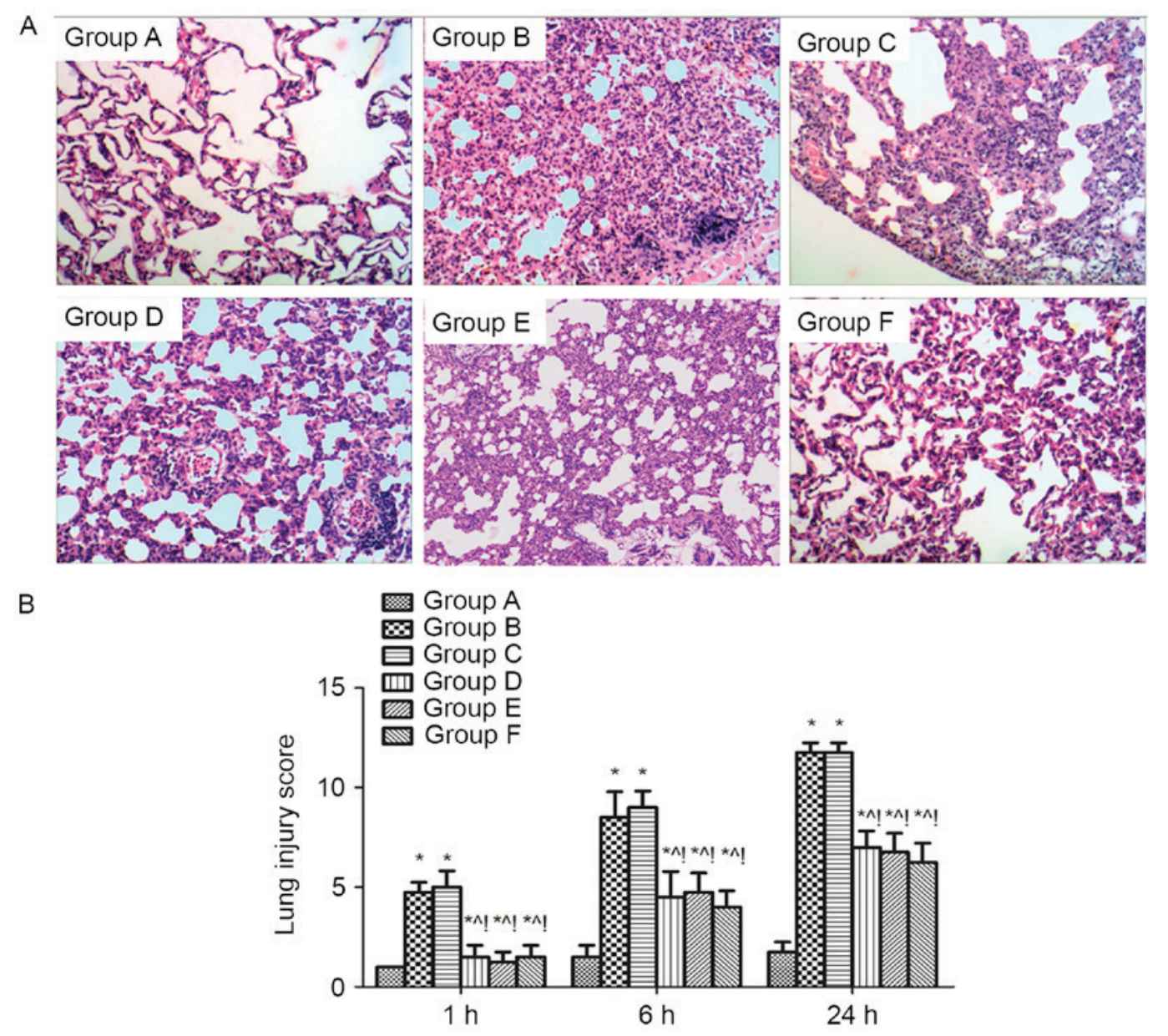

Figure 2. Effect of MAPK signalling on the histopathology of sepsis-induced lung injury. (A) Histopathological analysis of lung tissues was performed at 1, 6 and $24 \mathrm{~h}$ after the cecal ligation and puncture challenge. Magnification, x100. (B) Pathological lung injury scores were expressed as mean \pm standard deviation. The results showed a significant reduction in the severity of lung injury in group D, E and F mice compared with groups B and C. There was no difference between group B and $\mathrm{C} . \mathrm{n}=4$ at each time point for each group ${ }^{*} \mathrm{P}<0.05$ vs. group $\mathrm{A},{ }^{\wedge} \mathrm{P}<0.05$ vs. $\mathrm{B},{ }^{\mathrm{P}} \mathrm{P}<0.05$ vs. $\mathrm{C}, \mathrm{D}, \mathrm{E}$, and F. ARDS, acute respiratory distress syndrome; A, sham; B, ARDS; C, DMSO + ARDS; D, SP600125 + ARDS; E, SB203580 + ARDS; F, SP600125 + SB203580 + ARDS.

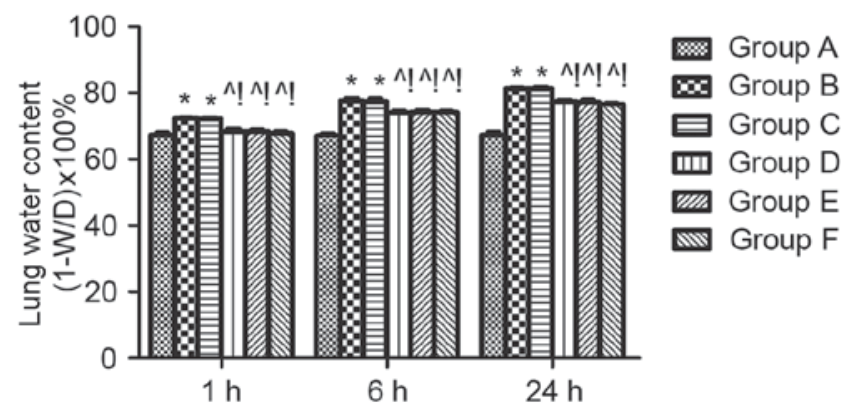

Figure 3. Effect of mitogen activated protein kinase signalling on sepsis-induced lung permeability. Lung edema was measured using the lung water content. W/D indicates the ratio of the lung wet weight to dry weight, which was detected at 1,6 and $24 \mathrm{~h}$ after cecal ligation and puncture. $\mathrm{n}=4$ at each time point for each group. The results are expressed as the mean \pm standard deviation. ${ }^{*} \mathrm{P}<0.05$ vs. group $\mathrm{A},{ }^{\wedge} \mathrm{P}<0.05$ vs. $\mathrm{B},{ }^{\mathrm{P}} \mathrm{P}<0.05$ vs. C, D, E, and F. ARDS, acute respiratory distress syndrome; A, sham; B, ARDS; C, DMSO + ARDS; D, SP600125 + ARDS; E, SB203580 + ARDS; F, SP600125 + SB203580 + ARDS.

Effect of MAPK signalling on the serum levels of inflammatory factors in sepsis-induced acute lung injury rats. The levels of the pro-inflammatory cytokines IL-6 and TNF- $\alpha$ and the anti-inflammatory cytokine IL-10 were measured in the serum of rats 1,6 , and $24 \mathrm{~h}$ after CLP challenge. Levels of all three cytokines were significantly higher at 6 , and $24 \mathrm{~h}$ when group B is compared with group A, while IL- 6 and TNF- $\alpha$ were increased at $1 \mathrm{~h}$ in group B compared with group A $(\mathrm{P}<0.05$; Fig. 4). IL-6 and TNF- $\alpha$ were reduced at 1,6 , and $24 \mathrm{~h}$ in the groups D, E and F compared with group B (Fig. 4A and $\mathrm{B})$, whereas IL-10 was increased $(\mathrm{P}<0.05$; Fig. $4 \mathrm{C})$. The same effects were found when compared with the DMSO control (group C) ( $\mathrm{P}<0.05$; Fig. 4).

\section{Discussion}

ARDS is regarded as part of a systemic inflammatory response, particularly systemic sepsis (24), which is usually accompanied by excessive inflammatory cell infiltration, cascade release of inflammatory factors, and extravasation of protein-rich fluid (25). Previous studies have determined that MAPK signaling pathways may have an important pathogenic role in the inflammatory process associated with sepsis-induced ARDS $(19,26,27)$. As the current study demonstrated, ARDS triggered by sepsis after CLP challenge may induce phosphorylation of JNK and p38 MAPK in the lung tissue and modulation of MAPK signaling by JNK or/and p38 MAPK-specific inhibitor 

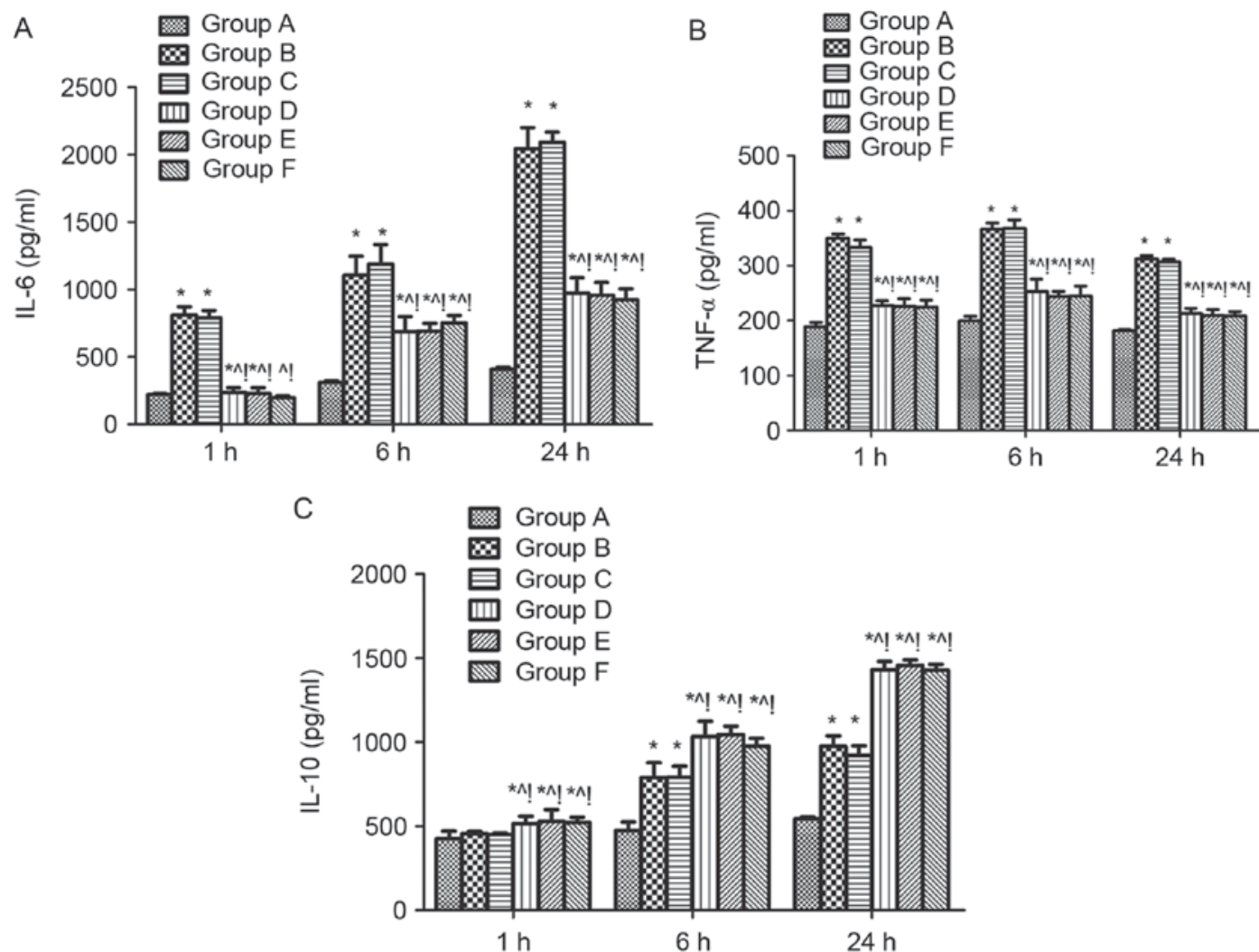

Figure 4. Effect of mitogen activated protein kinase signalling on the serum levels of inflammatory factors in sepsis-induced acute lung injury rats. Levels of the proinflammatory cytokines (A) IL-6, (B) TNF- $\alpha$ and anti-inflammatory cytokine (C) IL-10 in serum of rats 1, 6, and $24 \mathrm{~h}$ after cecal ligation and puncture challenge were measured using ELISA. $\mathrm{n}=4$ at each time point for each group. Data are expressed as the mean \pm standard deviation. "P<0.05 vs. group $\mathrm{A}$, ${ }^{\wedge} \mathrm{P}<0.05$ vs. B, 'P $<0.05$ vs. C, D, E, and F. IL-6, -10, interleukin-6, -10; TNF- $\alpha$, tumor necrosis factor- $\alpha$; ARDS, acute respiratory distress syndrome; A, sham; B, ARDS; C, DMSO + ARDS; D, SP600125 + ARDS; E, SB203580 + ARDS; F, SP600125 + SB203580 + ARDS.

may significantly improve the pulmonary histopathology and lung permeability, increasing the serum levels of anti-inflammatory factors and reducing the serum levels of pro-inflammatory factors in sepsis-induced acute lung injury rats.

Some of MAPKs, including ERK1, ERK2, p38 $\alpha$, JNK1 and JNK2 have been confirmed to be involved in innate immunity (10), Germline-encoded pattern recognition receptors (PRRs) recognize invariant microbial components, termed pathogen-associated molecular patterns and then stimulate the innate immune response after infection $(28,29)$. PRRs activate both MAPK and nuclear factor- $\kappa \mathrm{B}(\mathrm{NF}-\kappa \mathrm{B})$ pathways then activate the immune responses (30). Previous studies have revealed that in sepsis-induced ARDS mice after CLP the phosphorylation of p38 MAPK, ERK, and JNK increased significantly in lung tissue $(15,21)$. As the links between inflammation and ARDS (13), another study found that SB203580, a selective p38 inhibitor inhibited TNF production (14), and SP600125, a specific JNK inhibitor, reduced CLP-induced activation of JNK and modulated the early and late steps of the inflammatory cascade in a murine model of CLP-induced sepsis (31). According to these findings, the present study identified a robust phosphorylation of JNK and p38 MAPK in lung tissue of sepsis-induced ARDS rats after CLP challenge. The specific JNK or p38MAPK inhibitor, SP600125 or SB203580 administered by intragastric injection may significantly reduce the phosphorylation of JNK and p38 MAPK in lung tissues. This suggested that in sepsis-induced ARDS, the JNK and p38
MAPK signalling in lung tissue were stimulated, which could be downregulated by oral administration of their specific inhibitor.

Previous studies showed that inflammatory injuries trigger a robust influx of neutrophils and monocytes to the site of tissue injury (7) and the damaged or dead cells are thought to trigger the inflammasome-dependent responses, then alert the innate immune system to the impending tissue damage (8). Previous studies confirmed that the mechanism of some drugs, including Decitabine, 5-azacitidine, Losartan and Andrographolide sulfonate, protect lungs against injury induced by sepsis via the inhibition of the phosphorylation of the ERK, JNK and p38 MAPK, which may result in the suppression of the proinflammatory cytokine expression $(16,17,21)$. Inhibition of the p38 MAPK and JNK, but not ERK could alleviate inflammatory cell infiltration and microvascular permeability in sepsis-induced ARDS mice (32). It is possible that JNK and p38 MAPK have an important role in sepsis-induced lung injury. In the current study, lung injury after CLP increased the expression of phosphorylated JNK and p38MAPK in lung tissue and administration of the JNK and p38 MAPK inhibitor may be able to reduce the severity of lung injury in mice. The beneficial effects of the JNK and p38MAPK inhibitor achieved in this model confirmed that JNK and p38MAPK are essential for the development of ARDS after sepsis and the potential therapeutic effects of JNK and p38MAPK inhibitor in such pathological conditions were elucidated. 
A previous study determined that $\mathrm{p} 38$ MAPK activation was essential for CXCR3-mediated endothelial cell apoptosis and was associated with the increase of the leakage of protein-rich fluid and inflammatory cells in ARDS-induced lung by CLP (33). Inhibition of LPS-induced activation of JNK, ERK, and p38MAPK pathways in lung tissues may decrease the indices of pulmonary edema, lung wet-to-dry weight ratios markedly (19). Inhibition of the p38 MAPK and JNK, but not ERK may alleviate inflammatory cell infiltration and microvascular permeability in sepsis-induced ARDS mice (32). All of these findings suggested that the effect of JNK and p38 MAPK on sepsis-induced lung permeability. The present study found that after JNK and p38 MAPK inhibitor administration the lung edema in ARDS rats induced by sepsis after CLP was significantly improved, therefore it is possible that JNK and p38 MAPK were involved the deterioration of lung permeability following sepsis.

MAPKs also have an important role in inducing cytokine production. It has been previously established that inflammatory stimuli may lead to the activation of MAPK and the transcription factor $\mathrm{NF}-\kappa \mathrm{B}$, which mediates the expression of several pro-inflammatory cytokines, including TNF- $\alpha$, IL-1 $\beta$, and IL-6 which have an important role in many inflammatory disease processes (34-37). Previous studies indicated that $\mathrm{p} 38$ MAPK inhibitors can suppress IL- 6 and TNF- $\alpha$ expression in monocytes and mast cells $(38,39)$. Additional studies have suggested roles for $\mathrm{p} 38$ MAPK in the in vitro production of inflammatory factors, such as TNF- $\alpha$ (40), IL-6 (41), somewhat paradoxically, the anti-inflammatory factor IL-10 $(42,43)$. A previous study determined that the suppression of the activation of the JNK and p38 MAPK would significantly reduce TNF- $\alpha$ content in the plasma of LPS-induced ARDS mice (44). Another previous study revealed that berberine inhibited LPS-induced expression of proinflammatory genes including IL-1 $\beta$, IL-6 via suppression of the phosphorylation of p38, JNK and ERK in peritoneal macrophages (45). Hesperidin downregulated the LPS-induced expression of pro-inflammatory cytokines, including TNF- $\alpha$, IL-1 $\beta$, IL- 6 and enhanced the production of anti-inflammatory IL-10, IL-4, IL-12, which may be controlled by JNK and p38 MAPK pathways (46). Glutamine treatment inhibited phosphorylation of p38 MAPK and ERK pathways critical for cytokine release, meanwhile, significantly attenuated TNF- $\alpha$ and IL- 6 after CLP (47). A previous study found that the regulation of TLR4-mediated induction of TNF production is ERK1 and ERK2 independent (48). In the present study, the JNK and p38 MAPK inhibitor was able to significantly reduce the pro-inflammatory cytokines IL- 6 and TNF- $\alpha$. Meanwhile, an increase the anti-inflammatory cytokine IL-10 ws observed in the serum of rats after CLP challenge. It provides the direct evidence that the JNK and p38 MAPK have an important role in the system inflammation response induced by CLP in rats. This confirmed that JNK and p38 MAPK may be essential for the development of acute lung injury induced by sepsis.

It is of note noted that the present study did not identify a difference between the group D or E (single administration of SB203580 or SP600125) and the group F (combined application of SB203580 and SP600125) on pulmonary histopathology, lung permeability and the serum levels of inflammatory factors. This suggested that there may be a common downstream pathway between JNK and p38 MAPK and further investigation is required to confirm this.
In conclusion, JNK and p38 MAPK inhibitor improved the lung permeability, attenuated system inflammation and alleviated the lung injury induced by sepsis. JNK and p38 MAPK signaling are essential for the development of ARDS following sepsis, further investigations are required to elucidate the detailed mechanisms of JNK and p38 MAPK signaling in sepsis-induced ARDS.

\section{Acknowledgements}

The current study was supported by the Youth Scientific Research Foundation of the Affiliated Hospital of Qingdao University (grant no. 2384).

\section{References}

1. Ware LB and Matthay MA: The acute respiratory distress syndrome. N Engl J Med 342: 1334-1349, 2000.

2. Rubenfeld GD, Caldwell E, Peabody E, Weaver J, Martin DP, Neff M, Stern EJ and Hudson LD: Incidence and outcomes of acute lung injury. N Engl J Med 353: 1685-1693, 2005.

3. Goss CH, Brower RG, Hudson LD and Rubenfeld GD; ARDS Network: Incidence of acute lung injury in the United States. Crit Care Med 31: 1607-1611, 2003.

4. Hudson LD, Milberg JA, Anardi D and Maunder RJ: Clinical risks for development of the acute respiratory distress syndrome. Am J Respir Crit Care Med 151: 293-301, 1995.

5. Sevransky JE, Martin GS, Shanholtz C, Mendez-Tellez PA, Pronovost P, Brower R and Needham DM: Mortality in sepsis versus non-sepsis induced acute lung injury. Crit Care 13: R150, 2009.

6. de Luis Cabezon N, Sánchez Castro I, Bengoetxea Uriarte UX, Rodrigo Casanova MP, García Peña JM and Aguilera Celorrio L: Acute respiratory distress syndrome: A review of the Berlin definition. Rev Esp Anestesiol Reanim 61: 319-327, 2014 (In Spanish).

7. Kono $\mathrm{H}$ and Rock KL: How dying cells alert the immune system to danger. Nat Rev Immunol 8: 279-289, 2008.

8. Iyer SS, Pulskens WP, Sadler JJ, Butter LM, Teske GJ, Ulland TK, Eisenbarth SC, Florquin S, Flavell RA, Leemans JC and Sutterwala FS: Necrotic cells trigger a sterile inflammatory response through the Nlrp3 inflammasome. Proc Natl Acad Sci USA 106: 20388-932, 2009.

9. Strassheim D, Park JS and Abraham E: Sepsis: Current concepts in intracellular signaling. Int J Biochem Cell Biol 34: 1527-1533, 2002.

10. Arthur JS and Ley LC: Mitogen-activated protein kinases in innate immunity. Nat Rev Immunol 13: 679-692, 2013.

11. Kyriakis JM and Avruch J: Mammalian mitogen-activated protein kinase signal transduction pathways activated by stress and inflammation. Physiol Rev 81: 807-869, 2001.

12. Ahmed AU, Williams BR and Hannigan GE: Transcriptional Activation of Inflammatory Genes: Mechanistic insight into selectivity and diversity. Biomolecules 5: 3087-3111, 2015.

13. Cohen P: Targeting protein kinases for the development of anti-inflammatory drugs. Curr Opin Cell Biol 21: 317-324, 2009.

14. Dar AC and Shokat KM: The evolution of protein kinase inhibitors from antagonists to agonists of cellular signaling. Annu Rev Biochem 80: 769-795, 2011.

15. Kim SJ, Baek KS, Park HJ, Jung YH and Lee SM: Compound 9a, a novel synthetic histone deacetylase inhibitor, protects against septic injury in mice by suppressing MAPK signalling. Br J Pharmacol 173: 1045-1057, 2016.

16. Huang X, Kong G, Li Y, Zhu W, Xu H, Zhang X, Li J, Wang L, Zhang Z, Wu Y, et al: Decitabine and 5-azacitidine both alleviate LPS induced ARDS through anti-inflammatory/antioxidant activity and protection of glycocalyx and inhibition of MAPK pathways in mice. Biomed Pharmacother 84: 447-453, 2016.

17. Peng S, Hang N, Liu W, Guo W, Jiang C, Yang X, Xu Q and Sun Y: Andrographolide sulfonate ameliorates lipopolysaccharide-induced acute lung injury in mice by down-regulating MAPK and NF- $\kappa$ B pathways. Acta Pharm Sin B 6: 205-211, 2016.

18. Xu X, Liu N, Zhang YX, Cao J, Wu D, Peng Q, Wang HB and Sun WC: The protective effects of HJB-1, a derivative of 17-Hydroxy-Jolkinolide B, on LPS-induced acute distress respiratory syndrome mice. Molecules 21: 77, 2016. 
19. Kong G, Huang X, Wang L, Li Y, Sun T, Han S, Zhu W, Ma M, $\mathrm{Xu} \mathrm{H}$, Li J, et al: Astilbin alleviates LPS-induced ARDS by suppressing MAPK signaling pathway and protecting pulmonary endothelial glycocalyx. Int Immunopharmacol 36: 51-58, 2016.

20. Zhao H, Zhao M, Wang Y, Li F and Zhang Z: Glycyrrhizic acid prevents sepsis-induced acute lung injury and mortality in rats. J Histochem Cytochem 64: 125-137, 2016.

21. Shen L, Mo H, Cai L, Kong T, Zheng W, Ye J, Qi J and Xiao Z: Losartan prevents sepsis-induced acute lung injury and decreases activation of nuclear factor kappaB and mitogen-activated protein kinases. Shock 31: 500-506, 2009.

22. Otero-Antón E, González-Quintela A, López-Soto A,López-Ben S, Llovo J and Pérez LF: Cecal ligation and puncture as a model of sepsis in the rat: Influence of the puncture size on mortality, bacteremia, endotoxemia and tumor necrosis factor alpha levels. Eur Surg Res 33: 77-79, 2001.

23. Mrozek JD, Smith KM, Bing DR, Meyers PA, Simonton SC Connett JE and Mammel MC: Exogenous surfactant and partial liquid ventilation: Physiologic and pathologic effects. Am J Respir Crit Care Med 156: 1058-1065, 1997.

24. Dolinay T, Kim YS, Howrylak J, Hunninghake GM, An CH, Fredenburgh L, Massaro AF, Rogers A, Gazourian L, Nakahira K, et al: Inflammasome-regulated cytokines are critical mediators of acute lung injury. Am J Respir Crit Care Med 185: 1225-1234, 2012.

25. Matthay MA, Zimmerman GA, Esmon C, Bhattacharya J, Coller B, Doerschuk CM, Floros J, Gimbrone MA Jr, Hoffman E, Hubmayr RD, et al: Future research directions in acute lung injury: summary of a National Heart, Lung and Blood Institute working group. Am J Respir Crit Care Med 167: 1027-1035, 2003.

26. Wu H, Zhao G, Jiang K, Chen X, Zhu Z, Qiu C, Li C and Deng G Plantamajoside ameliorates lipopolysaccharide-induced acute lung injury via suppressing NF- $\mathrm{KB}$ and MAPK activation. Int Immunopharmacol 35: 315-322, 2016.

27. Lee JH, Ko HJ, Woo ER, Lee SK, Moon BS, Lee CW, Mandava S, Samala M, Lee J and Kim HP: Moracin M inhibits airway inflammation by interrupting the JNK/c-Jun and NF- $\kappa$ B pathways in vitro and in vivo. Eur J Pharmacol 783: 64-72, 2016.

28. Newton K and Dixit VM: Signaling in innate immunity and inflammation. Cold Spring Harb Perspect Biol 4: pii: a006049, 2012.

29. Kawai $\mathrm{T}$ and Akira S: The role of pattern-recognition receptors in innate immunity: Update on Toll-like receptors. Nat Immunol 11: 373-384, 2010

30. Medzhitov R and Horng T: Transcriptional control of the inflammatory response. Nat Rev Immunol 9: 692-703, 2009.

31. Pizzino G, Bitto A, Pallio G, Irrera N, Galfo F, Interdonato M Mecchio A, De Luca F, Minutoli L, Squadrito F and Altavilla D: Blockade of the JNK signalling as a rational therapeutic approach to modulate the early and late steps of the inflammatory cascade in polymicrobial sepsis. Mediators Inflamm 2015: 591572, 2015.

32. Zhou H, Bian D, Jiao X, Wei Z, Zhang H, Xia Y, He Y and Dai Y: Paeoniflorin protects against lipopolysaccharide-induced acute lung injury in mice by alleviating inflammatory cell infiltration and microvascular permeability. Inflamm Res 60: 981-990, 2011

33. Zhu X, Zou Y, Wang B, Zhu J, Chen Y, Wang L, Li J and Deng X: Blockade of CXC chemokine receptor 3 on endothelial cells protects against sepsis-induced acute lung injury. J Surg Res 204 288-296, 2016

34. Rahman A and Fazal F: Blocking NF- $\kappa$ B: An inflammatory issue. Proc Am Thorac Soc 8: 497-503, 2011.
35. McKenna $S$ and Wright $C J$ : Inhibiting I $\kappa B \beta-N F \kappa B$ signaling attenuates the expression of select pro-inflammatory genes. J Cell Sci 128: 2143-2155, 2015.

36. Lee IT and Yang CM: Inflammatory signalings involved in airway and pulmonary diseases. Mediators Inflamm 2013: 791231, 2013

37. Lai JL, Liu YH, Liu C, Qi MP, Liu RN, Zhu XF, Zhou QG, Chen YY, Guo AZ and Hu CM: Indirubin Inhibits LPS-induced inflammation via TLR4 abrogation mediated by the NF-kB and MAPK signaling pathways. Inflammation 40: 1-12, 2017.

38. Guo X, Gerl RE and Schrader JW: Defining the involvement of p38alpha MAPK in the production of anti- and proinflammatory cytokines using an SB 203580-resistant form of the kinase. J Biol Chem 278: 22237-22242, 2003.

39. Jeong HJ, Na HJ, Hong SH and Kim HM: Inhibition of the stem cell factor-induced migration of mast cells by dexamethasone. Endocrinology 144: 4080-4086, 2003.

40. Schafer PH, Wang L, Wadsworth SA, Davis JE and Siekierka JJ: T cell activation signals up-regulate $\mathrm{p} 38$ mitogen-activated protein kinase activity and induce TNF-alpha production in a manner distinct from LPS activation of monocytes. J Immunol 162: 659-568, 1999 .

41. Beyaert R, Cuenda A, Vanden Berghe W, Plaisance S, Lee JC, Haegeman G, Cohen P and Fiers W: The p38/RK mitogen-activated protein kinase pathway regulates interleukin- 6 synthesis response to tumor necrosis factor. EMBO J 15: 1914-1923, 1996

42. Foey AD, Parry SL, Williams LM, Feldmann M, Foxwell BM and Brennan FM: Regulation of monocyte IL-10 synthesis by endogenous IL-1 and TNF-alpha: Role of the p38 and p42/44 mitogen-activated protein kinases. J Immunol 160: 920-928, 1998.

43. Koprak S, Staruch MJ and Dumont FJ: A specific inhibitor of the p38 mitogen activated protein kinase affects differentially the production of various cytokines by activated human T cells: Dependence on CD28 signaling and preferential inhibition of IL-10 production. Cell Immunol 192: 87-95, 1999.

44. Yu X, Yu S, Chen L, Liu H, Zhang J, Ge H, Zhang Y, Yu B and Kou J: Tetrahydroberberrubine attenuates lipopolysaccharide-induced acute lung injury by down-regulating MAPK, $\mathrm{AKT}$, and NF- $\mathrm{BB}$ signaling pathways. Biomed Pharmacother 82: 489-497, 2016.

45. Jeong HW, Hsu KC, Lee JW, Ham M, Huh JY, Shin HJ, Kim WS and Kim JB: Berberine suppresses proinflammatory responses through AMPK activation in macrophages. Am J Physiol Endocrinol Metab 296: E955-E964, 2009.

46. Yeh CC, Kao SJ, Lin CC, Wang SD, Liu CJ and Kao ST: The immunomodulation of endotoxin-induced acute lung injury by hesperidin in vivo and in vitro. Life Sci 80: 1821-1831, 2007.

47. Singleton KD, Beckey VE and Wischmeyer PE: Glutamine prevents activation of NF-kappaB and stress kinase pathways, attenuates inflammatory cytokine release, and prevents acute respiratory distress syndrome (ARDS) following sepsis. Shock 24: 583-589, 2005.

48. Yang HT, Papoutsopoulou S, Belich M, Brender C, Janzen J, Gantke T, Handley M and Ley SC: Coordinate regulation of TPL-2 and NF- $\kappa \mathrm{B}$ signaling in macrophages by NF- $\mathrm{KB} 1 \mathrm{p} 105$. Mol Cell Biol 32: 3438-3451, 2012. 